\title{
Self-reciprocal fermion mass ratios from massless QED with curved momentum space
}

\author{
B.E.J. Bodmann ${ }^{a, *}$, Th.A.J. Maris ${ }^{b}$ \\ ${ }^{a}$ Centro de Ciências Exatas e Tecnológicas, Universidade do Vale do Rio dos Sinos, São Leopoldo, RS, Brazil \\ ${ }^{\mathrm{b}}$ Instituto de Física, Universidade Federal do Rio Grande do Sul, Porto Alegre, RS, Brazil
}

Received 23 March 2000; received in revised form 10 October 2000; accepted 13 October 2000

Editor: M. Cvetič

\begin{abstract}
The present investigation is an attempt to understand the fermion mass ratios in the framework of QED of charged fermions without a bare mass. Since QED of massless charged fermions is invariant under the dilatation transformation, this symmetry has to be spontaneously broken to obtain massive fermions. In the proposed model we combine a mass-scale normalisation with the renormalisation procedure, assuming the fermion momentum space being a four-dimensional one-shell hyperboloid embedded in a five-dimensional space. The hyperboloid constrains the allowed fermion field solutions. We construct the theory in the conventional way using equal time anti-commutator and the Lagrangian formalism. Starting from the Dyson-Schwinger equation for fermion propagator in the Landau gauge, we derive the fermion mass function and self-reciprocal solutions for the mass ratios, which are independent of any constant. @ 2000 Published by Elsevier Science B.V.
\end{abstract}

Keywords: Curved energy-momentum space; Fermion mass ratios

\section{Introduction}

One of the still outstanding questions is the understanding of the nature of fermion mass ratios of the elementary particles. The Standard Model (for a review see [1-3]), which takes into account the three small scale relevant interactions, up to now holds quite well against experimental tests, so that one might hope to calculate the masses of the elementary particles on this basis [4-7]. However, the model does not offer any clue on how to reduce the parameter set of experimentally determined values in order to genuinely shed light on the origin of the elementary particle masses.

\footnotetext{
* Corresponding author.

E-mail address: bardo@exatas.unisinos.tche.br (B.E.J. Bodmann).
}

A remarkable fact is that in physical theories measurable quantities are in general expressed in units of length. One has however always the freedom to choose the unit of length which is used. A change of this unit transforms the values of all quantities according to their dimension, but neither changes the formalism nor the value of any meaningful physical observable, as these are dimensionless. This suggests that a fundamental symmetry of a basic theory should be the dilatational one, but that this invariance is spontaneously broken and therefore admits a continuous set of descriptions, each of which may be characterised by the arbitrary choice of a unit of length.

In Quantum Field Theory it is an accepted practice in renormalisation prescriptions to introduce a dimensional scale into the theory, thence, it is no longer sufficient to specify the Lagrangian density, but also 
the scale parameter and consequently the theory is no longer scale invariant. It appears therefore an attractive idea to understand the scale parameter on a rather natural basis, i.e., as a consequence of a spontaneously broken dilatation invariance. The scope of the present discussion is to test the hypothesis of spontaneously broken dilatation symmetry, which is valid if self-reciprocal fermion mass ratios may be found from a massless theory. Hence, we combine the always necessary mass-scale normalisation with the renormalisation procedure in a simple model of charged massless leptons. We assume that the original theory is defined in a 5-dimensional space and has a generalised Lorentz and dilatation invariance. No scale of length dimension is fixed in the original theory with its five dimensions. There is supposed to exist a spontaneous dilatation symmetry breaking which reduces the physical momentum-space to a four-dimensional one, consisting of a connected hyper-surface $H$ embedded in a five-dimensional space. It turns out that a description with such a vacuum is possible and remains quite similar to the usual four-dimensional space-time formalism of fermion fields but with a relativistically invariant momentum cutoff. We point out, that we do not change the usual description of the space-time manifold carrying the photon field. There is no quantisation into separate points or any other structural change of space-time. The role of the momentum hyper-surface, to be introduced, will only be to put a symmetry condition on the allowed solutions of the fermion field equations and belongs in this sense to these equations.

The rotation and dilatation in the original 5-dimensional momentum-space should give a complete manifold of different but physically equivalent symmetry relations of the 5-dimensional field theory. Each of these solutions should correspond to a certain choice for the unit of length. Of course, it would be ideal to start from the formalism of the 5-dimensional theory and to derive how a spontaneous symmetry breaking creates such a manifold instead of imposing it. Several trials have shown us that such a theory might well exist, but we have not yet found a satisfactory solution. However, even the assumption that there exists a 5dimensional momentum space theory, which allows an infinite set of symmetry breaking solutions with the 4dimensional vacua of our present model seems to lead to interesting conclusions and we hope to find the original 5-dimensional theory at a later time. The simple model of charged massless leptons on the mentioned momentum hyper-surface may then be considered a by intuition motivated first approach, which nevertheless is self-consistent as shown by the results.

\section{Field theory on a momentum hyperboloid}

First we consider the space-time symmetries of the classical field theory of a charged massless fermion field. Besides being gauge invariant such a theory is invariant under the $S O(4,2)$-group of conformal transformations, formed by the Poincaré subgroup, spacetime reflections dilatations and the special conformal transformations. Since special conformal transformations may mix space- and time-like vectors, they are not compatible with a Quantum Field Theory [8]. Therefore the space-time symmetries of the QED of massless fermions, which we shall discuss, consists only of the Poincaré transformations, reflections and dilatations. In the momentum space this subgroup consists of the local phase transformation, Lorentz transformation, reflections and dilatations.

As we remarked, in nature the dilatation invariance is broken, but the Lorentz transformations are conserved. We shall start from momentum space instead from space-time. The Lorentz transformations in momentum space have the usual form and we shall define the theory of our model in a way to be translationally invariant in space-time coordinates. Even in the very special case of a massless QED it would be desirable to specify the basic theory and to show how its overall dilatation symmetry is spontaneously broken, giving the residual symmetries of the vacuum which we shall postulate in the following. It appears that our present assumptions are not self contradictory and might indicate the direction of the more basic theory.

As an initial approach we restrict our discussion to the equation of the lowest order self-energy diagram. We admit that it is difficult to formally justify the exclusion of weak as well as strong interactions in higher order diagrams, that include all kinds of vacuum polarisation terms and show for the simplified case, how a model seems to allow an approximation to observed considerably large mass ratios, which contains as the only parameter the electromagnetic coupling constant. To this end, we assume that the four-dimensional energy-momentum space for fermions is a curved one, 
namely consisting of the surface of an one-shell hyperboloid $H$, embedded in a five-dimensional space with the usual coordinates $p_{0}, p_{1}, p_{2}, p_{3}$ and $p_{5}$ as an extra one, which is the simplest possible five-dimensional momentum space geometry in agreement with invariance under combined dilatation and Lorentz transformation [9].

In the following the physical momenta have index $\mu=0, \ldots, 3$ and the index $\alpha$ refers to $0, \ldots, 3$ and 5. $H$ is defined by the relation: $\widehat{M}^{2}=g_{\alpha \beta} p^{\alpha} p^{\beta}=$ $p_{5}^{2}+p_{0}^{2}-p_{1}^{2}-p_{2}^{2}-p_{3}^{2}=M^{2}$. Here, $M$ signifies the massive vacuum state selected by the spontaneously broken symmetry fixing an arbitrary but positive mass scale. Due to its origin this value should not enter in any measurable quantity. The coordinates of a unique point on $H$ may be chosen to be $p_{\mu}$ and $\iota=p_{5} /\left|p_{5}\right|=$ \pm 1 . $H$ is invariant under the five-dimensional unitary transformation with the generators

$$
\begin{gathered}
M_{\alpha}^{\beta}=i\left(p_{\alpha} \frac{\partial}{\partial p_{\beta}}-p^{\beta} \frac{\partial}{\partial p^{\alpha}}\right), \\
\text { with }\left[M_{\alpha}^{\beta}, M_{\beta}^{\gamma}\right]=i g_{\beta}^{\beta} M_{\alpha}^{\gamma},
\end{gathered}
$$

where the $M_{\mu}^{\nu}$ 's correspond to the usual space-time Lorentz rotations, and in the commutation relation no sum over $\beta$ is understood. Note, that the generator's commutators in Eq. (1) obey besides linearity and antisymmetry the Jacobi identity. From the remaining $M_{5}^{v}$ operations, we define the space-time operators in momentum-space representation:

$$
\begin{aligned}
x^{\mu} & =\frac{1}{2}\left\{p_{5}^{-1}, M_{5}^{\mu}\right\} \\
& =\lim _{\epsilon \rightarrow 0} i\left(\frac{\partial}{\partial p_{\mu}}-\frac{1}{2}\left\{\left(p_{5} \sqrt{1+\frac{\epsilon^{2}}{p_{5}^{2}}}\right)^{-1}, \frac{\partial}{\partial 5}\right\} p^{\mu}\right),
\end{aligned}
$$

where $\{\cdot, \cdot\}$ denotes the anti-commutator, and we have regularised the expression $1 / p_{5}$. These operators commute with each other and with $p_{\alpha} p^{\alpha}$ and have the usual commutation rules with $M_{\mu}^{v}$ and $p_{\mu}$. In contradistinction to other investigations $[10,11]$ our configuration space is from the start four- and not fivedimensional. The different role of $p_{5}$ from the one of $p_{0}$ implies symmetry breaking in the original 5dimensional theory.

Considering the Lorentz invariance of $p_{5}$ it is also of interest to introduce the iso-spin-like operator, which connects the two shell halves with $\operatorname{sign}\left(p_{5}\right)= \pm 1$ and $t_{3}=\operatorname{sign}\left(p_{5}\right) \delta^{4}\left(p_{\mu}-p_{\mu}^{\prime}\right), t_{1}=\delta\left(p_{5}+p_{5}^{\prime}\right) \delta^{4}\left(p_{\mu}-\right.$ $\left.p_{\mu}^{\prime}\right), t_{2}=i t_{1} t_{3}$. These operators on $H$ obey the usual iso-spin relations and commute with $x_{\mu}$ and $p_{\mu}$. The operator set $\widehat{M}, x_{\mu}$ and $t_{3}$ is complete for the fivedimensional $p_{\alpha}$-space. A set of eigenfunctions is given by

$$
\begin{array}{r}
\left\langle p_{\alpha} \mid x_{\mu}=x_{\mu}^{\prime} ; t_{3}= \pm 1 ; \widehat{M}=M\right\rangle \\
=\lim _{\epsilon \rightarrow 0} \frac{1}{4 \pi^{2}} \theta\left( \pm p_{5}\right) \sqrt{\frac{p_{5}^{2}+\epsilon^{2}}{M^{2}}} \\
\quad \times e^{-i p_{\mu} x^{\mu}} \delta\left(M-\sqrt{p_{\alpha} p^{\alpha}}\right),
\end{array}
$$

with $\left\langle x^{\prime},{ }_{j}, M^{\prime} \mid x, l, M\right\rangle=\delta^{4}\left(x_{\mu}-x_{\mu}^{\prime}\right) \delta\left(M-M^{\prime}\right) \delta_{l \jmath}$. Our basic assumption is that all fermion fields can have only momenta located on an hyperboloid $H$, with a definite value of $M$ for $\widehat{M}$ and we shall only consider operators which commute with $\widehat{M}$, i.e., keep $M$ invariant, in agreement with the assumption of a spontaneously broken scale invariance. At this point we introduce an artifice, which simplifies the calculations without affecting the results. To avoid expressions of the type $\delta\left(M-M^{\prime}\right)$, one would have to consider arbitrarily narrow probability distributions of $M$ values around the value $M^{\prime}$ and to integrate these in each operation. This complicates the formalism without adding anything new and we therefore take the mean value of the possible eigenvalues of $\widehat{M}$, which form an arbitrarily dense spectrum. The $\delta\left(M-M^{\prime}\right)$ operator reduces then to $\delta_{M M^{\prime}}$, for operators with one $M$-value. In the framework of spontaneously broken symmetries, $M$ defines the vacuum corresponding to the broken dilatation invariance and serves therefore as a mass unit. In the limit of $M \rightarrow \infty$, with $p_{\mu}$ finite, $p_{5}$ simultaneously goes to infinity, which leaves us with the conventional massless four-momentum theory. Additionally the twofoldness looses its original meaning as the $p_{5}$-positive and negative halves of the hyperboloid become disconnected. In the four-dimensional hyperboloid space the set of $x_{\mu}$-eigenfunctions (2) is not anymore orthogonal, as the integration over the $M$-values is missing. Because of the identity

$$
\begin{aligned}
& \int_{-M}^{M} \frac{p_{5}}{M} \delta\left(M-\sqrt{p_{\alpha} p^{\alpha}}\right) \delta\left(M^{\prime}-\sqrt{p_{\alpha} p^{\alpha}}\right) d p_{5} \\
& \quad=\delta\left(M-M^{\prime}\right) \theta\left(M^{2}-p_{\mu} p^{\mu}\right)
\end{aligned}
$$


which now becomes $\delta_{M M^{\prime}} \theta\left(M^{2}-p_{\mu} p^{\mu}\right)$, one obtains on $H$ from relation (3)

$$
\begin{aligned}
& \left\langle p_{\mu}, t_{3}=\imath \mid x_{\mu}^{\prime} t_{3}=J\right\rangle \\
& \qquad=(2 \pi)^{-2} \theta\left(M^{2}-p_{\mu} p^{\mu}\right) e^{-i p_{\mu} x^{\mu}} \delta_{l \jmath} \\
& \text { and }\left\langle x_{\mu}^{\prime}, \imath \mid x_{\mu}\right\rangle=Q\left(x^{\prime}-x\right) \delta_{l \jmath} \\
& \text { with } \quad Q\left(x^{\prime}-x\right) \\
& \quad=(2 \pi)^{-2} \int e^{i p_{\mu}\left(x_{\mu}-x_{\mu}^{\prime}\right)} \theta\left(M^{2}-p_{\mu} p^{\mu}\right) d^{4} p .
\end{aligned}
$$

$Q$ is a measure for the expression of the lepton localisation in space-time and substitutes the usual $\delta^{4}(x-$ $\left.x^{\prime}\right)$ function with which it has the following features in common: Symmetry under $x \leftrightarrow x^{\prime}$ exchange, idempotency, further it recovers the delta functional in the limit of flat momentum space and $\int Q\left(x-x^{\prime}\right) d x_{0}=$ $\int Q\left(x-x^{\prime}\right) d x_{0}^{\prime}=\delta^{3}\left(\vec{x}-\vec{x}^{\prime}\right) . Q$ is a projection operator on the linear set of two component wavefunctions (the constituents of the mass ratio) with a Lorentz invariant four-momentum cutoff $M$, which was to be expected from our hyperboloid definition. The basic advantage of the five-dimensional approach over the two component direct cutoff formalism is, that the inclusion of the coordinate $p_{5}$ into Lorentz invariant expressions yields a natural analyticity of the cutoff procedure and further connects between the two field components, which may be characterised by their $t_{3}$ eigenvalues.

We have made the basic assumption that the charged fermion fields can have only field components in the space defined by Eq. (3), with fixed $M$. The momenta are in this case, for free fermions with mass $m$ given by the two curves with $p_{5}= \pm \sqrt{M^{2}-m^{2}}$ on the four-dimensional hyperboloid. Thus we can construct the theory in the conventional way, starting from equal-time anti-commutators and the usual Lagrangian and Hamiltonian formalism are valid. The only new effect is that we have a two component field, corresponding to the two signs of $p_{5}$. An essential deviation from the conventional theory becomes apparent if one considers fermions interacting with the electromagnetic field, because in the $H$-space no virtual fermion momenta larger than $M$ are allowed. We take as usual the Dirac-Lagrangian density but assume for $\Psi(x)$ and its allowed variations the condition $Q \Psi_{i}(x)=\int Q\left(x-x^{\prime}\right) \Psi_{i}\left(x^{\prime}\right) d^{4} x^{\prime}=\Psi_{i}(x)$ with $i= \pm$, which is the hyperboloid hypothesis. This con- dition does not contradict the usual equal-time anticommutation rules, which we assume to be true. Seen from the point of view of the usual formulation of field theory, our assumption implies that the Lehmanndensity of the lepton propagator vanishes for invariant momentum values exceeding $M$. A gauge transformation may be performed by using the freedom of choosing a factor $\exp \{i \phi(x)\}$ in the basic set of (3) of eigenfunctions of the $x^{\mu}$ s defined in Eq. (2). In that function space the four-momentum is represented by $\left(i \frac{\partial}{\partial x^{\mu}}-\frac{\partial \phi}{\partial x^{\mu}}\right)$, which may as usual be interpreted as a change of $A^{\mu}(x)$. Consequently, there is no contradiction or alteration to the established gauge theory of quantum fields with four-dimensional momentum space, except for the necessary momentum cutoff that enters through momentum-space curvature.

One may develop the operator field theory in the common way, for instance, using the interaction representation with the interaction given by Eq. (6) and for the Feynman propagator of the fermion one has relation (7)

$$
\begin{aligned}
& S_{\text {int }}= \int \bar{\Psi}_{i}(x) \mathcal{A}(x) \Psi_{i}(x) d x \\
& \equiv \int\left(\bar{\Psi}_{i} Q\right)(x) \mathcal{A}(x)\left(Q \Psi_{i}\right)(x) d x, \\
& S_{i j}\left(x-x^{\prime}\right)= \int Q\left(x^{\prime}-y^{\prime}\right) S_{i j}\left(y^{\prime}-y\right) \\
& \times Q(y-x) d^{4} y d^{4} y^{\prime} .
\end{aligned}
$$

The photon propagator remains the original one and the vertex is modified by the projection $Q$ for each fermion leg, which by virtue prevents forbidden momentum transfers.

\section{The Dyson-Schwinger equation}

One may derive the Dyson-Schwinger equation for the Fermion propagator with the help of Feynman diagrams, in the usual way. We take as approximation the bare vertex, using the Landau gauge, then the $\not p$ component of the self-energy vanishes (Refs. $[3,12,13])$. Further the vacuum polarisation only is taken into account by using the experimental coupling constant $e$. Summing the relevant perturbation series one finds for 
the mass function,

$$
\begin{aligned}
& Q(p) m_{ \pm}(p)=-i \frac{e^{2}}{(2 \pi)^{4}} Q(p) \\
& \quad \times \int \frac{1}{\left(p-p^{\prime}\right)^{2}} Q\left(p^{\prime}\right) \frac{m_{ \pm}\left(p^{\prime}\right)}{p^{\prime 2}-m_{ \pm}^{2}\left(p^{\prime}\right)} d^{4} p^{\prime} .
\end{aligned}
$$

Except for the projection operators $Q$, this is the common result for a two component fermion field. Applying for $\left|p^{2}\right|<M^{2}$ the operator $\frac{\partial}{\partial p_{\mu}} \frac{\partial}{\partial p^{\mu}}$, one obtains for this range the differential equation $(s=$ $\left.p_{0}^{2}-\underline{p}^{2}\right)$ :

$$
\begin{gathered}
Q(+s)\left(s \frac{d^{2}}{d s^{2}}+2 \frac{d}{d s}\right) m_{ \pm}(s) \\
=-\frac{3 e^{2}}{(4 \pi)^{2}} Q(+s) \frac{m_{ \pm}}{s-m_{ \pm}^{2}}
\end{gathered}
$$

$\left(Q(+s)=\theta\left(M^{2}-p_{0}^{2}+\underline{p}^{2}\right)\right)$. These are still the usual equations (Ref. [14]), except for the cutoff of $s=$ $p_{0}^{2}-\underline{p}^{2}$ at $M^{2}$. Due to the hyperboloid hypothesis for any given $p_{5}$ there is an estimated upper limit for a possible mass pole, since $m^{2} \ll M^{2}$. In fact, the higher $\left|p_{5}\right|<M$ the lower the mass pole limit becomes, and in the limit $M \rightarrow \infty$ the mass pole tends to zero. Thus for $M<\infty$ any two massive solutions $m_{ \pm}$are connected through the boundary condition at $s=M^{2}$ or equivalently $p_{5}=0$.

The differential equation (9) may be transformed in a polynomial equation for the mass with the help of Cauchy's integral theorem for a closed path in the complex mass-plane around the mass pole of the order $\lambda$, resulting in

$$
\begin{aligned}
0= & -\frac{\beta}{2} \sqrt{s}+4 b_{2 \lambda+1 \mid \lambda}+2 c_{2 \lambda+1 \mid \lambda} \\
& +2 m_{ \pm}^{2} c_{2 \lambda+2 \mid \lambda} \\
b_{\kappa \mid \lambda}= & \sum_{\nu=0}^{\kappa}(\nu-\lambda)(\kappa-v-\lambda) a_{\nu} a_{\kappa-\nu}, \\
c_{\kappa \mid \lambda}= & \sum_{\nu=0}^{\kappa}(v-\lambda)(\nu-\lambda-1)(\kappa-v-\lambda) a_{\nu} a_{\kappa-\nu},
\end{aligned}
$$

where the coefficients $b_{\kappa \mid \lambda}$ and $c_{\kappa \mid \lambda}$ depend on the Laurent expansion coefficients $a_{v}$ of the mass function around the mass pole. Note, that the appearance of $\sqrt{s}$ in Eq. (10) is a manifestation of the branch cut. Upon integrating $s$ out with $m_{ \pm}^{2} \leqslant s \leqslant M^{2}$ and using the fact that $c_{2 \lambda+2 \mid \lambda} \equiv 0$, yields for the real mass solutions,

for $\lambda=1$ :

$\left|m_{ \pm}\right|=\frac{M}{\sqrt{2}} \sqrt{-1+\xi^{2}+\sqrt{3 \mp 8 \xi-6 \xi^{2}+4 \xi^{4}}}$,

for $\lambda \geqslant 2$ :

$$
\begin{aligned}
& \left|m_{ \pm}\right| \\
& =\frac{M}{\sqrt{2}} \sqrt{-1+\xi^{2}+\sqrt{\left(1-\xi^{2}\right)^{2}-4\left(1 \mp 2 \xi+\xi^{2}\right)}},
\end{aligned}
$$

which permits to calculate the mass ratios (see Fig. 1), where we have tacitly absorbed $\beta$ and the $a_{\nu} \mathrm{s}$ in one variable $\xi$. The resulting mass ratio function $R(\xi)$ yields considerably large mass ratios for $\lambda=1$, whereas for $\lambda \geqslant 2$ the permitted range, i.e., where the mass solution is real valued is of the order of unity. As may be seen from Fig. 1, the regions where large mass ratios occur is the interval symmetrically around $\xi=0$ as indicated by a dark bar. For $\lambda \geqslant 2$ only the semi-open intervals (light bars) allow for real valued solutions. For any mass ratio with valid $\xi$ the reciprocal mass ratio exists for $-\xi$, which reflects the before mentioned $t_{3}$ operation. As example the self-reciprocal mass ratio solutions $\left(R_{x y}(\xi)=R_{x y}^{-1}(-\xi)\right)$, for the charged leptons $e, \mu$ and $\tau$ are given, $R_{\mu e}(\xi=-0.225531)=206.77=$ $R_{\mu e}^{-1}(\xi=0.225531), R_{\tau e}(\xi=-0.225537)=3491.4$ $=R_{\tau e}^{-1}(\xi=0.225537)$ and $R_{\tau \mu}(\xi=-0.224727)=$ $16.89=R_{\tau \mu}^{-1}(\xi=0.224727)$.

\section{Discussion and remarks}

We are completely aware of the fact, that our model at the present status is restricted to the question whether spontaneous dilatation symmetry breaking in a curved momentum space may be a candidate for an explication of the existent mass ratios. Our reasoning is far from leading to a theory, which is applicable to the complete set of elementary particles and their interactions. Nevertheless, the hyperbolic momentum configuration space of constant curvature with its large mass parameter $M$ already shows its physical effect, i.e., the considerable large mass ratios, which we regard as the meaningful result of our approach. 


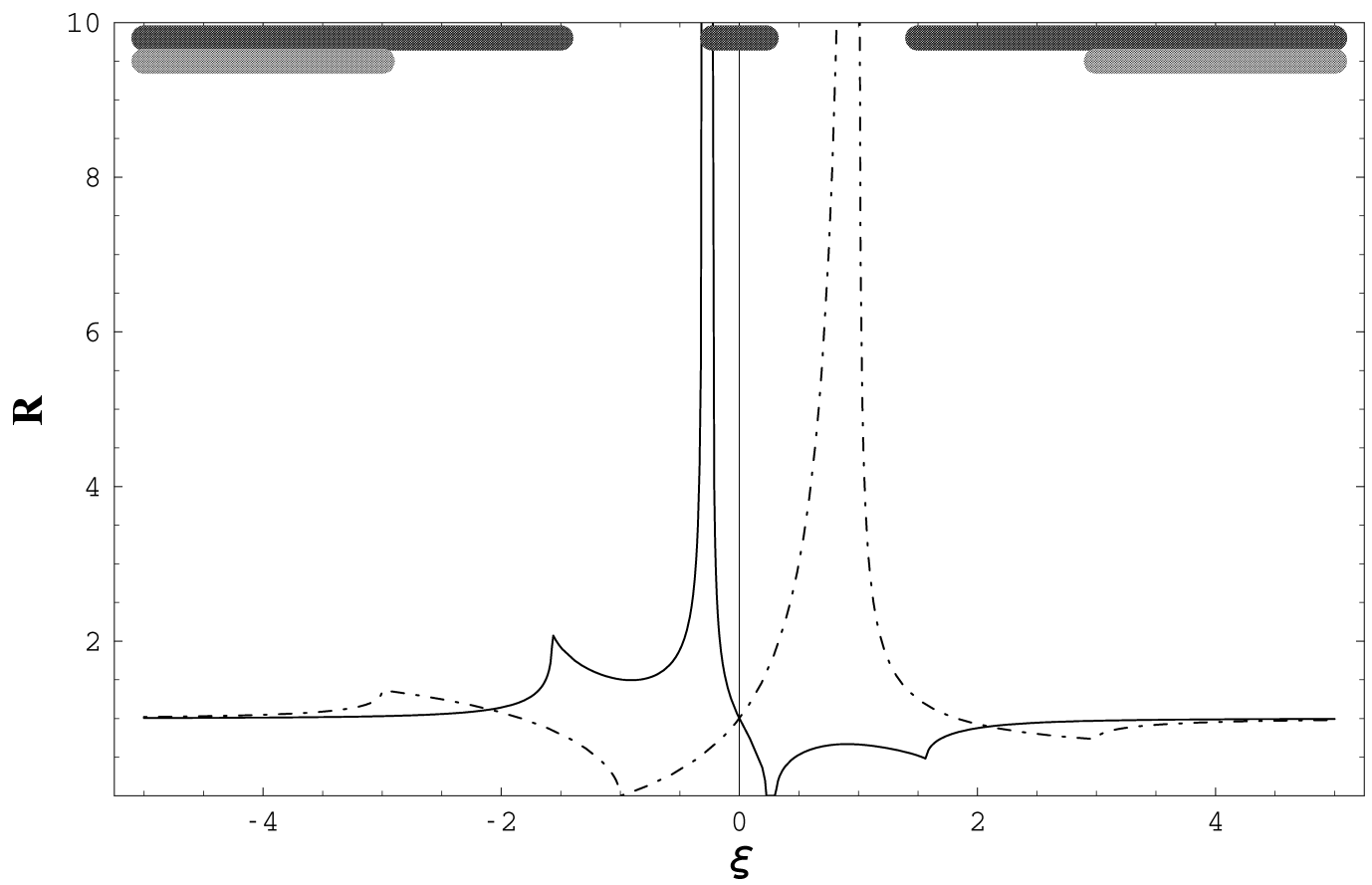

Fig. 1. The self-reciprocal fermion mass ratios $R(\xi)=R^{-1}(-\xi)$ are determined for $\lambda=1$ (solid line) and for $\lambda \geqslant 2$ (dot-dashed line). The permitted range with a real valued mass ratio is indicated by a dark bar for $\lambda=1$ and a light bar for $\lambda \geqslant 2$.

Evidently our treatment, leaves many questions open: We have among others neglected the vacuum polarisation in the photon propagator. As extremely high momenta play an important role, the influence of this approximation should be investigated. In Ref. [14] the vacuum polarisation was judged to be essential, but that discussion is not applicable to our case. We have used the Landau gauge and the important question of gauge invariance, which we have only briefly mentioned and should be worked out in detail for our case. Further, only constant momentum space curvature and QED has been taken into account. One further step will therefore be to examine how $M$ and the hyperboloid are affected if non-Abelian gauge symmetry structures are taken into account in a more general theory.

The Goldstone theorem might seem to require in QED with zero bare fermion mass a pseudo-scalar zero mass boson, because of the spontaneous $\gamma_{5}$ symmetry breaking, however, similar problems occur in certain versions of QCD and several ways to circumvent it there have been proposed. To us, a nat- ural way to do this in the present case is to realise that the conserved formal $\gamma_{5}$-current is a highly singular quantity, which has to be renormalised. In this self-consistent procedure functionals of the interacting fields enter and these carry the spontaneously broken symmetry properties. This renormalisation process may destroy the exact current conservation. In this way not only a symmetry but with it the corresponding current conservation can be spontaneously broken. In other words, in certain spontaneously broken symmetry cases Noether's theorem may not be valid and consequently Goldstone's theorem not applicable (see, for instance, [15]).

In the case of a spontaneously broken symmetry in general more descriptions of the theory are possible, each based on a different vacuum and related by the symmetry transformation, but all giving the same values for observables. In our case, where the dilatation invariance, the $\gamma_{5}$ invariance and the $p_{5}$ reflection $\left(=\tau_{3}\right)$ symmetry are spontaneously broken, we found indeed the freedom to choose $M$, to change the signs of both masses, and to change the mass ratio 
from $R$ to $R^{-1}$. It is remarkable that our result does not depend on any constant of nature. But an essential question is now whether a better treatment of the mass equation would give a discrete mass spectrum, which will be a topic of future investigation together with the question, whether a generalisation of the present approach, for instance to a more sheeted hyperboloid like structure is possible, in order to somehow organise the fermion generations.

\section{References}

[1] E. Leader, E. Predazzi, An Introduction to Gauge Theories and Modern Particle Physics, Cambridge Monographs on Particle Physics, Nuclear Physics and Cosmology, Cambridge Univ. Press, 1996.

[2] F. Scheck, Electroweak and Strong Interactions, SpringerVerlag, Berlin, Heidelberg, New York, 1996.
[3] S. Weinberg, The Quantum Theory of Fields, Vol. II, Cambridge Univ. Press, 1996.

[4] D. Akers, Int. J. Theor. Phys. 33 (7) (1994) 1523.

[5] K.S. Babu, S.R. Barr, Phys. Lett. B 381 (1996) 137.

[6] M. Calixto, V. Aldaya, J. Phys. A 32 (1999) 7287.

[7] C.D. Froggatt, H.B. Nielsen, D.J. Smith, Phys. Lett. B 385 (1996) 150

[8] R.E. Marshak, Conceptual Foundations of Modern Particle Physics, World Scientific, Singapore, New Jersey, London, 1993, pp. 138-143.

[9] J. Zinn-Justin, Quantum Field Theory and Critical Phenomena, Oxford Univ. Press, Oxford, New York, Toronto, 1993, pp. 296-298.

[10] V.G.K. Kadyshevsky, D.V. Fursaev, Theor. Math. Phys. 83 (1990) 197

[11] R.M. Mir-Kasimov, Preprint: International Center for Theoretical Physics IC/90/407 (1990).

[12] M. Hirayama, Prog. Theor. Phys. 82 (2) (1989) 396.

[13] Th.A.J. Maris, G. Jacob, Phys. Rev. Lett. 17 (26) (1966) 1300.

[14] R. Fukuda, K. Kugo, Nucl. Phys. B 117 (1976) 250.

[15] Th.A.J. Maris et al., Nucl. Phys. B 37 (1972) 444 